\title{
Urinary phthalate metabolites in relation to maternal serum thyroid and sex hormone levels during pregnancy: a longitudinal analysis
}

Lauren E Johns' ${ }^{1}$ Kelly K Ferguson' ${ }^{1}$, Offie P Soldin², David E Cantonwine ${ }^{3}$, Luis O Rivera-González', Liza V Anzalota Del Toro ${ }^{4}$, Antonia M Calafat ${ }^{5}$, Xiaoyun Ye ${ }^{5}$, Akram N Alshawabkeh ${ }^{6}$, José F Cordero ${ }^{4}$ and John D Meeker ${ }^{1 *}$

\begin{abstract}
Background: Increasing scientific evidence suggests that exposure to phthalates during pregnancy may be associated with an elevated risk of adverse reproductive outcomes such as preterm birth. Maternal endocrine disruption across pregnancy may be one pathway mediating some of these relationships. We investigated whether urinary phthalate metabolites were associated with maternal serum thyroid (free thyroxine [FT4], free triiodothyronine [FT3], and thyroid-stimulating hormone [TSH]), and sex (estradiol, progesterone, and sex hormone-binding globulin [SHBG]) hormone levels at multiple time points during pregnancy.
\end{abstract}

Methods: Preliminary data $(n=106)$ were obtained from an ongoing prospective birth cohort in Northern Puerto Rico. We collected urine and serum sample at the first and third study visits that occurred at $18+/-2$ and $26+/-$ 2 weeks of gestation, respectively. To explore the longitudinal relationships between urinary phthalate metabolites and serum thyroid and sex hormone concentrations, we used linear mixed models (LMMs) adjusted for prepregnancy body mass index (BMI) and maternal age. An interaction term was added to each LMM to test whether the effect of urinary phthalate metabolites on serum thyroid and sex hormone levels varied by study visit. In cross-sectional analyses, we stratified BMI- and age-adjusted linear regression models by study visit.

Results: In adjusted LMMs, we observed significant inverse associations between mono-3-carboxypropyl phthalate (MCPP) and FT3 and between mono-ethyl phthalate (MEP) and progesterone. In cross-sectional analyses by study visit, we detected stronger and statistically significant inverse associations at the third study visit between FT3 and MCPP as well as mono-carboxyisooctyl phthalate (MCOP); also at the third study visit, significant inverse associations were observed between FT4 and metabolites of di-(2-ethylhexyl) phthalate (DEHP). The inverse association between MEP and progesterone was consistent across study visits.

Conclusions: In this group of pregnant women, urinary phthalate metabolites may be associated with altered maternal serum thyroid and sex hormone levels, and the magnitude of these effects may depend on the timing of exposure during gestation.

Keywords: Biomarkers, Endocrine disruption, Epidemiology, Thyroid, Hormones, Urinary metabolites, Pregnancy, Phthalates

\footnotetext{
* Correspondence: meekerj@umich.edu

${ }^{1}$ Department of Environmental Health Sciences, University of Michigan

School of Public Health, Ann Arbor, MI, USA

Full list of author information is available at the end of the article
} 


\section{Background}

Phthalate diesters are commonly used as plasticizers in industrial applications [1]. Additionally, many household and consumer products, including flooring and wall coverings, food packaging, and cosmetics such as lotions and fragrances, contain phthalates [2-4]. Due to their ubiquitous use, human exposure to phthalates is widespread [5]. Because phthalates are not chemically bound to the plastics and other products that contain them, they can easily leach into household dust, food and water sources, and ambient air $[1,6,7]$. Consequently, human exposure to phthalates can occur through ingestion, inhalation, or dermal absorption [1,3].

Growing evidence suggests that urinary concentrations of phthalate metabolites during pregnancy are associated with adverse reproductive outcomes including preterm birth and pregnancy loss $[8,9]$. Hormonal production and regulation are critical for pregnancy maintenance and fetal growth and neurodevelopment; maternal endocrine disruption during pregnancy may be one pathway mediating some of these relationships [10-13].

Limited human health studies have examined the potential thyroid-altering effects of phthalates. In a cross-sectional study of men recruited from a U.S. fertility clinic, the urinary concentration of mono-(2-ethylhexyl) phthalate (MEHP), a metabolite of di-(2-ethylhexyl) phthalate (DEHP), was inversely associated with free thyroxine (3, 3, 5, 5'-tetraiodoL-thyronine, FT4) and total triiodothyronine (3, 3', 5-triiodo-L-thyronine, T3) [14]. Urinary concentrations of DEHP metabolites were also inversely associated with total T3 and total and free T4, and positively associated with thyroid-stimulating hormone (thyrotropin, TSH) in a representative sample of U.S. adults (non-pregnant women and men) participating in the National Health and Nutritional Examination Survey (NHANES) [15]. Similar inverse relationships between urinary DEHP metabolites and total and free T3 (FT3) were reported in a cross-sectional study of Danish children [16]. While pervasive exposure to phthalates has been documented among pregnant women worldwide [17-22], human health studies investigating the effects of phthalate exposure during pregnancy on maternal thyroid function are scarce. Huang and colleagues [23] reported an inverse association between urinary concentrations of monobutyl phthalate (MBP), the metabolite of dibutyl phthalate (DBP), and both free and total T4 in the second trimester of Taiwanese pregnant women.

Animal and in vitro studies have also shown that phthalates can interfere with sex hormone concentrations, signaling, and/or function [24,25], which may profoundly affect implantation, fetal development, and parturition $[10,26]$. However, human data pertaining to the relationships between phthalates and sex hormones are limited. In men recruited through a U.S. fertility clinic, urinary MEHP was inversely associated with serum estradiol levels
[27]. A positive association between urinary MEHP and sex hormone-binding globulin (SHBG) was also reported in separate cohort of fertile U.S. men [28]. In a recent study conducted among pregnant women in the U.S., an inverse association was found between urinary DEHP metabolite concentrations and serum testosterone concentrations, while no statistically significant relationship was observed for estradiol [29]. Additionally, Hart and coauthors [30] reported significantly inverse correlations between serum MEHP and SHBG concentrations at both 18 weeks and 36 weeks of gestation among pregnant women in Australia.

We are aware of no published investigations that longitudinally evaluate the potential thyroid-disrupting effects of environmental phthalate exposure among pregnant women. Furthermore, whether alterations in thyroid and sex hormone levels vary by time point of exposure during gestation remains largely undetermined and may have important influences on downstream hormonemediated reproductive outcomes. In this preliminary analysis, we investigated the relationship between urinary phthalate metabolites and maternal serum thyroid (FT3, FT4, and TSH) and sex (estradiol, progesterone, and SHBG) hormone levels measured in samples collected at two time points in pregnancy from women participating in a prospective birth cohort in Puerto Rico.

\section{Methods \\ Study population}

The Puerto Rico Testsite for Exploring Contamination Threats (PROTECT) project is an ongoing prospective birth cohort in the Northern Karst Region of Puerto Rico designed to investigate the relationship between phthalates and other environmental contaminants and adverse pregnancy outcomes such as preterm birth. A previous study within this project reported greater concentrations of some urinary phthalate metabolites measured in Puerto Rican participants compared to women of reproductive age in the contiguous U.S. [31]. The current analysis included data collected from the first 106 pregnant women participating in the PROTECT project with urinary phthalate metabolites and serum thyroid and sex hormones measures completed as of November 2012. Study participants, aged 18 to 40 years, were recruited around $14 \pm 2$ weeks gestation from 7 prenatal clinics and hospitals throughout Northern Puerto Rico from 2010 to 2012. Participant recruitment and eligibility criteria as well as sample collection and processing are described in detail elsewhere [31]. Demographic information was obtained from questionnaires administered at the initial study visit. Spot urine samples were collected from each participant at three separate study visits (visit 1: $18 \pm 2$ weeks, visit 2: $22 \pm 2$ weeks, visit 3: $26 \pm 2$ weeks of gestation). Only urine samples from visits 1 and 3 were utilized in the present 
analyses because blood samples were not collected at visit 2. All participants in the present analysis provided urine and serum samples for at least one visit (visits 1 and/or 3). Upon collection and processing (e.g., aliquoting, centrifugation, and separation of blood into plasma and serum components), all urine and blood samples were frozen at $-80^{\circ} \mathrm{C}$ until shipped overnight on dry ice to the analytical laboratories where samples were again stored at $-80^{\circ} \mathrm{C}$ until analysis.

The study protocols were approved by the ethics and research committees of the participating institutions. The involvement of the Centers for Disease Control and Prevention $(\mathrm{CDC})$ laboratory was determined not to constitute engagement in human subjects research. The study was described in detail to all participating women and all study participants gave informed consent.

\section{Measurement of phthalate metabolites}

Available urine samples ( $\mathrm{N}=196$ samples, $\mathrm{N}=106$ participants) were analyzed by the Centers for Disease Control and Prevention (CDC) laboratories, using protocols developed for NHANES, to measure urinary concentrations (free plus glucuronidated) of 11 phthalate metabolites: MEHP, MBP, mono-2-ethyl-5-hydroxyhexyl phthalate (MEHHP), mono-2-ethyl-5-oxohexyl phthalate (MEOHP), mono-2-ethyl-5-carboxypentyl phthalate (MECPP), mono3-carboxypropyl phthalate (MCPP), mono-carboxyisooctyl phthalate (MCOP), mono-carboxyisononyl phthalate (MCNP), mono-benzyl phthalate (MBzP), mono-iso-butyl phthalate (MiBP), and mono-ethyl phthalate (MEP). The analytical method involved enzymatic deconjugation of the metabolites from their glucuronidated form, solidphase extraction, separation by high-performance liquid chromatography, and detection by isotope-dilution tandem mass spectrometry [32]. The limits of detection (LOD) were in the low nanogram per milliliter range [31]. Concentrations below the LOD were assigned a value of LOD divided by the square root of 2 [33]. To account for urine dilution, urinary specific gravity (SG) was measured using a digital handheld refractometer (Atago Co., Ltd., Tokyo, Japan). For descriptive analyses, metabolite concentrations were standardized using the following formula: $\mathrm{P}_{\mathrm{SG}}=\mathrm{P}[(1.019-1) /(\mathrm{SG}-1)]$, where $\mathrm{P}_{\mathrm{SG}}$ is the specific gravity-adjusted phthalate metabolite concentration $(\mathrm{ng} / \mathrm{mL}), \mathrm{P}$ is the observed phthalate metabolite concentration, 1.019 was the specific gravity population median, and SG is the specific gravity of the urine sample.

\section{Measurement of thyroid hormones}

For 106 subjects, serum samples from visits $1(\mathrm{~N}=106)$ and $3(\mathrm{~N}=89)$ were available for measurement of FT3, FT4, and TSH at the Bioanalytical Core Laboratory of Georgetown University (Washington, DC). Limitations in sample volume contributed to differences in the number of samples per study visit available for analysis. Following an ultrafiltration step to separate the free hormones, FT3 and FT4 were measured using isotope dilution liquid chromatography tandem mass spectrometry per the methods described in detail previously [34-37]. TSH concentrations were measured using a solid-phase immunochemiluminometric assay (DPC Immulite, Diagnostic Products Corporation) according to the manufacturer's instructions.

\section{Measurement of sex hormones}

For 104 subjects, serum samples were analyzed for estradiol (visit 1: $\mathrm{N}=103$ samples; visit 3: $\mathrm{N}=86$ samples) and progesterone (visit $1: \mathrm{N}=104$; visit $3: \mathrm{N}=89$ ) using a chemiluminescence immunoassay (DPC Immulite, Diagnostic Products Corporation). Serum levels of SHBG from samples collected from 99 subjects (visit 1 : $N=88$; visit 3 : $\mathrm{N}=69$ ) were determined using the same procedures. Similar to the thyroid hormone analyses, limitations in sample volume contributed to differences in available samples for each sex hormone/study visit. These analyses were also performed by the Bioanalytical Core Laboratory at Georgetown University (Washington, DC).

\section{Statistical analysis}

Serum concentrations of FT4, FT3, estradiol, and SHBG closely approximated normality and were untransformed in statistical analyses. TSH, progesterone, and urinary phthalate metabolite concentrations were positively skewed and were logarithmically transformed prior to analyses. Because MEHP, MEOHP, MEHHP, and MECPP share a single parent compound, the sum of concentrations of the DEHP metabolites ( $\Sigma$ DEHP) was calculated from the molar sum $(\mathrm{nmol} / \mathrm{mL})$ of these four metabolites and logtransformed in statistical analyses. Means and standard deviations as well as selected percentiles were used to examine the distributions of the urinary phthalate metabolites, thyroid hormones, and sex hormones. Geometric means and standard deviations were calculated for nonnormally distributed variables.

Pearson correlations were calculated to assess the relationships between continuous variables. To test the differences in the mean concentrations of sex and thyroid hormones by visit, we used linear mixed models (LMMs) with serum hormones levels regressed on study visit and included a random intercept for subject ID to account for intra-individual correlation of repeated measurements over time. Urinary phthalate metabolites and sex and thyroid hormones were tested for associations with demographic variables to examine potential confounding. We used LMMs with one phthalate metabolite concentration measure (as exposure) and one outcome per model to explore the longitudinal relationships between urinary phthalate metabolites and sex and thyroid hormone 
concentrations. Crude models were adjusted for urinary specific gravity and study visit. Full models additionally included maternal prepregnancy body mass index (BMI) and age, both measured at the initial study visit. BMI was modeled as a categorical variable $\left(\leq 25 \mathrm{~kg} / \mathrm{m}^{2},>25\right.$ and $\leq 30 \mathrm{~kg} / \mathrm{m}^{2},>30 \mathrm{~kg} / \mathrm{m}^{2}$ ) and age was modeled as a continuous variable. BMI and age were included as covariates because of their potential influence on urinary phthalate metabolite concentrations [38] and sex and thyroid hormone levels [39-41]. A likelihood ratio test for fixed effects was used to identify additional potential covariates in full models. An interaction term was added to each full LMM to test whether the effect of urinary phthalate metabolites on thyroid and sex hormone serum levels varied by study visit.

In a secondary analysis, we used linear regression models with one phthalate metabolite concentration and one outcome per model to investigate the cross-sectional relationships between urinary phthalate metabolites and sex and thyroid hormone serum concentrations at each study visit (visits 1 and 3). These models were also adjusted for maternal BMI, age, and urinary specific gravity. To enhance interpretability, all regression coefficients and associated 95\% confidence intervals (CIs) generated from the LMMs and linear regression models were expressed as the percent change in hormone serum levels for an interquartile range (IQR) increase in urinary phthalate metabolite concentrations. Associations were considered statistically significant at the $5 \%$ level and marginally significant at the $10 \%$ level. All statistical analyses were conducted using SAS version 9.2 (SAS Institute Inc., Cary, NC).

\section{Results}

Table 1 shows the distribution of sociodemographic characteristics of the 106 pregnant women included in the present analysis. The study participants were generally highly educated ( $79.2 \%$ had at least a college education), and did not smoke during pregnancy (94.3\%). Approximately half $(55.7 \%)$ of the women had a prepregnancy BMI $\leq 25 \mathrm{~kg} / \mathrm{m}^{2}$. No statistically significant differences were observed between the demographic characteristics of study participants with measurable thyroid and sex hormone concentrations at visit 1 vs. those with measurable thyroid and sex hormone concentrations at visit 3 (chi-square test p-values $>0.05$ ). Based on the eligibility criteria, no participants used oral contraceptives within three months prior to pregnancy or underwent in vitro fertilization as a method of assisted reproductive technology, and all participants were free of known medical or obstetric complications.

The distributions of the 11 urinary phthalate metabolite concentrations across pregnancy have been previously investigated in this cohort [31]. Greater than $90 \%$ of the measured concentrations of all 11 urinary phthalate metabolites
Table 1 Sample characteristics of 106 pregnant women participating in PROTECT project (2010-2012)

\begin{tabular}{|c|c|}
\hline Variable & Mean \pm SD or $n(\%)$ \\
\hline Maternal age at enrollment (years) & $27.1 \pm 4.8$ \\
\hline \multicolumn{2}{|l|}{ Maternal education } \\
\hline Missing & $3(2.8)$ \\
\hline$<$ High school & $12(11.3)$ \\
\hline High school/equivalent & $7(6.6)$ \\
\hline College & $84(79.2)$ \\
\hline \multicolumn{2}{|l|}{ Annual household income (US\$) } \\
\hline Missing & $17(16.0)$ \\
\hline$\$ 20,000$ & $45(42.4)$ \\
\hline$\geq \$ 20,000$ to $<\$ 50,000$ & $27(25.5)$ \\
\hline$\geq \$ 50,000$ & $17(16.0)$ \\
\hline \multicolumn{2}{|l|}{ Race } \\
\hline Missing & $3(2.8)$ \\
\hline White & $52(49.1)$ \\
\hline Mixed & $39(36.8)$ \\
\hline Other & $12(11.3)$ \\
\hline \multicolumn{2}{|l|}{ Prepregnancy BMI $\left(\mathrm{kg} \mathrm{m}^{-2}\right)$} \\
\hline Missing & $4(3.8)$ \\
\hline$\leq 25$ & $59(55.7)$ \\
\hline$>25$ to $\leq 30$ & $31(29.2)$ \\
\hline$>30$ & $12(11.3)$ \\
\hline \multicolumn{2}{|l|}{ Smoked during pregnancy } \\
\hline Missing & $5(4.7)$ \\
\hline Yes & $1(0.9)$ \\
\hline No & $100(94.3)$ \\
\hline \multicolumn{2}{|c|}{ Alcohol use at first visit during pregnancy } \\
\hline Missing & $4(3.8)$ \\
\hline Yes & $12(11.3)$ \\
\hline No & $90(84.9)$ \\
\hline
\end{tabular}

PROTECT, Puerto Rico Testsite for Exploring Contamination Threats, SD, standard deviation, BMI, body mass index.

were detectable [31]. Concentrations for a majority of the metabolites measured were greater in the Puerto Rican participants when compared to women of reproductive age in the contiguous U.S. [31]. For example, the geometric mean concentration of urinary MEHP (unadjusted for urinary dilution) was more than twice as high in the Puerto Rican cohort than the corresponding concentration found in women of reproductive age participating in the 20092010 NHANES study ( 3.3 vs. $1.6 \mathrm{ng} / \mathrm{mL}$, respectively) [31]. Spearman correlations between the majority of urinary metabolites were modest $(R=0.23-0.45)$, while strong correlations were observed between all urinary metabolites of DEHP (MEHP, MEHHP, MEOHP, and MECPP; $R>0.83$ ) [31]. With the exception of MCOP, no statistically 
significant differences were observed in the geometric mean concentrations between study visits for any of the other urinary phthalate metabolites (Additional file 1: Table S1).

The distributions of the thyroid and sex hormones are presented in Table 2. All of the measured concentrations of the hormones were detectable. We found a significantly positive Pearson correlation between estradiol and progesterone $(R=0.66, p<0.001)$. Weak but significant positive correlations were observed between FT4 and FT3 $(\mathrm{R}=0.17, \mathrm{p}=0.02)$, and between SHBG and both progesterone $(R=0.23, p=0.004)$ and estradiol $(R=0.29$, $\mathrm{p}=0.0002)$. Age was inversely correlated with thyroid and sex hormone levels (Additional file 2: Table S2). With the exception of TSH, LMMs showed that thyroid hormone levels differed significantly by study visit (Table 3). We found significantly higher mean levels of serum FT3 and FT4 at visit 1 compared to corresponding levels at visit 3. Conversely, the mean serum levels of all three sex hormones were significantly lower at visit 1 compared to visit 3.

Table 4 shows the fully adjusted longitudinal associations between urinary phthalate metabolite concentrations and serum hormones concentrations from LMMs. Each model contained random intercepts only for subject ID to account for intra-individual correlation, as addition of random slopes did not improve the model fit. A likelihood ratio test for fixed effects indicated that maternal education did not have a significant effect on serum hormone levels. Thus, prepregnancy BMI, maternal age, study visit, and urinary specific gravity were the only covariates retained in the final models. The crude regression results were similar to the adjusted results (not shown). We detected a significant inverse relationship between MCPP and FT3, where an IQR increase in MCPP was associated with a $2.89 \%$ decrease in FT3 $(95 \% \mathrm{CI}=-5.65$ to -0.02 , $\mathrm{p}=0.049$ ). We also observed a significant inverse association between MEP and progesterone. An IQR increase in
MEP was associated with a $10.6 \%$ decrease in progesterone $(95 \% \mathrm{CI}=-17.6$ to $-2.84, \mathrm{p}=0.01)$. We detected no significant associations between serum hormones levels and $\Sigma$ DEHP urinary metabolites in longitudinal analyses. Similarly, null results were observed for individual DEHP metabolites, with the exception of a marginally significant inverse association found between MEHHP and SHBG (percent change in outcome with IQR increase $[\% \Delta]=-4.62,95 \% \mathrm{CI}=-10.0$ to $0.75, \mathrm{p}=0.09)$. Most urinary phthalate metabolites were positively associated with TSH, although none of these relationships were statistically significant. We also found no statistically significant associations for FT4 or estradiol in the longitudinal analyses.

In our examination of the potential interaction between study visit and urinary phthalate metabolite concentrations in longitudinal analyses, we observed that study visit significantly modified the relationships between FT3 and MBzP $(\mathrm{p}=0.03)$ and MCPP $(\mathrm{p}=0.03)$, between FT4 and MiBP $(\mathrm{p}=0.01)$ and $\Sigma$ DEHP metabolites $(\mathrm{p}=$ $0.048)$, and between TSH and MBP ( $\mathrm{p}=0.04)$. We found no statistically significant interactions between study visit and urinary phthalate metabolites for any of the sex hormones examined (data not shown).

Table 5 presents the results from cross-sectional analyses by study visit for associations between urinary phthalate metabolites and serum thyroid hormone concentrations. At visit 1 , we observed a statistically significant positive association between MiBP and FT4 $(\% \Delta=4.95,95 \% \mathrm{CI}=0.27$ to $9.28, \mathrm{p}=0.04)$. MBzP and $\Sigma \mathrm{DEHP}$ metabolites were also positively, though not significantly, associated with FT4 at visit 1 . At visit 3 , urinary phthalate metabolites were generally inversely associated with FT4. At this visit, we found a statistically significant inverse association between $\Sigma$ DEHP and FT4 (\% $\Delta=-8.02,95 \% \mathrm{CI}=-15.3$ to $-0.80, \mathrm{p}=0.03)$. Urinary phthalate metabolites were generally positively associated with TSH at each study visit, and suggestive associations were observed with MBzP at visit 1 and MEP

Table 2 Distributions of serum hormone concentrations ( $N=106$ pregnant women)

\begin{tabular}{|c|c|c|c|c|c|c|c|}
\hline \multirow[t]{2}{*}{ Biomarker } & \multirow[t]{2}{*}{$\mathbf{N}$} & \multirow{2}{*}{$\begin{array}{c}\text { Mean } \\
\text { (SD) }\end{array}$} & \multicolumn{5}{|c|}{ Selected percentiles } \\
\hline & & & 25th & 50th & 75th & 95th & Max. \\
\hline \multicolumn{8}{|l|}{ Thyroid hormones } \\
\hline TSH (ulU/mL)† & 195 & $1.13(1.62)$ & 0.81 & 1.10 & 1.51 & 2.83 & 4.29 \\
\hline Free T3 (pg/mL) & 195 & $3.94(0.60)$ & 3.50 & 3.90 & 4.40 & 4.90 & 5.70 \\
\hline Free T4 (ng/dL) & 195 & $1.39(0.32)$ & 1.20 & 1.40 & 1.50 & 1.90 & 3.10 \\
\hline \multicolumn{8}{|l|}{ Sex hormones* } \\
\hline Progesterone $(\mathrm{ng} / \mathrm{mL}) \dagger$ & 193 & $62.8(1.60)$ & 45.7 & 57.3 & 82.0 & 140 & 374 \\
\hline Estradiol (ng/mL) & 189 & $8.16(4.06)$ & 4.79 & 7.58 & 11.8 & 15.0 & 15.0 \\
\hline SHBG (nmol/L) & 157 & 368 (109) & 293 & 352 & 428 & 563 & 736 \\
\hline
\end{tabular}

SD, standard deviation, TSH, thyroid-stimulating hormone, SHBG, sex hormone-binding globulin.

†Geometric mean and geometric standard deviation reported.

*Limitations in sample volume contributed to differences in available samples for each sex hormone. 
Table 3 Mean serum hormone concentrations (SD) by visit during gestation ( $\mathrm{N}=106$ pregnant women)

\begin{tabular}{cccc}
\hline Biomarker & $\begin{array}{c}\text { Visit 1 } \\
\text { (14-20 weeks) }\end{array}$ & $\begin{array}{c}\text { Visit 3 } \\
(\mathbf{2 4 - 2 8} \text { weeks) }\end{array}$ & p-value* \\
\hline Thyroid Hormones & & & \\
TSH (ulU/mL)† & $1.10(1.60)$ & $1.17(1.65)$ & 0.25 \\
Free T3 (pg/mL) & $4.01(0.61)$ & $3.86(0.59)$ & 0.02 \\
Free T4 (ng/dL) & $1.45(0.31)$ & $1.32(0.32)$ & 0.001 \\
Sex Hormones & & & \\
Progesterone (ng/mL)† & $46.9(1.37)$ & $88.4(1.48)$ & $<0.001$ \\
Estradiol (ng/mL) & $5.70(2.48)$ & $11.1(3.60)$ & $<0.001$ \\
SHBG (nmol/L) & $349(108)$ & $391(106)$ & 0.002 \\
\hline
\end{tabular}

SD, standard deviation, TSH, thyroid-stimulating hormone, SHBG, sex hormone-binding globulin.

tGeometric mean and geometric standard deviation reported.

*Test for fixed effects from linear mixed models with random intercepts.

at visit 3. While FT3 was inversely associated with both $\mathrm{MCPP}$ and MCOP at visit 1, these effects were greater and statistically significant at visit 3 .

The results of cross-sectional analyses for the relationships between urinary phthalate metabolites and sex hormone concentrations are presented in Table 6. We observed a marginally significant inverse relationship between MEP and SHBG at visit $1(\% \Delta=-10.1,95 \% \mathrm{CI}=-21.8$ to $1.65, \mathrm{p}=0.09$ ), and a null association at visit 3 . The inverse association between MEP and progesterone observed in the longitudinal analyses appeared consistent across study visits.

\section{Discussion}

To our knowledge the present study is the first to examine the relationships between urinary phthalate metabolites and serum thyroid hormone levels longitudinally across pregnancy. Few associations were observed in longitudinal analyses, although we did find statistically significant inverse associations between FT3 and MCPP, a non-specific metabolite of several high molecular weight phthalate plasticizers, and also between progesterone and MEP, the main metabolite of diethyl phthalate commonly used in personal care products. We also found that study visit of sample collection significantly modified the relationships between certain urinary phthalate metabolites and thyroid hormone levels. Our cross-sectional analyses corroborated our findings from the interaction analyses, and showed that associations between certain urinary phthalate metabolites and FT3 and FT4 hormone levels differed markedly (in terms of both magnitude and significance) based on visit of sample collection. For visit 1, associations with FT3 and FT4 were generally inverse, but at visit 3 these inverse relationships were stronger. Taken together, these results suggest that phthalate exposure during pregnancy may alter maternal thyroid and sex hormone levels. They also suggest that the magnitude of the potential

Table 4 Longitudinal analysis: percent change $(95 \% \mathrm{Cls})$ in thyroid and sex hormone concentrations in relation to interquartile range increase in urinary phthalate metabolite concentration

\begin{tabular}{|c|c|c|c|c|c|c|}
\hline \multirow[t]{2}{*}{ Analyte } & $\%$ Change (95\% Cl) & p-value & $\%$ Change (95\% Cl) & p-value & $\%$ Change $(95 \% \mathrm{Cl})$ & $p$-value \\
\hline & \multicolumn{2}{|c|}{ Free T3 ( $n=181$ observations) } & \multicolumn{2}{|c|}{ Free T4 ( $n=181$ observations) } & \multicolumn{2}{|c|}{ In-TSH (n = 181 observations) } \\
\hline $\mathrm{MBz} P$ & $0.18(-3.42,3.77)$ & 0.92 & $0.58(-4.45,5.56)$ & 0.82 & $5.51(-4.40,16.5)$ & 0.28 \\
\hline MBP & $1.33(-1.90,4.67)$ & 0.42 & $0.63(-4.00,5.21)$ & 0.79 & $3.28(-5.58,12.8)$ & 0.48 \\
\hline MiBP & $0.19(-2.86,3.14)$ & 0.90 & $1.14(-3.06,5.42)$ & 0.59 & $4.64(-3.73,13.0)$ & 0.28 \\
\hline MEP & $1.11(-2.74,4.96)$ & 0.57 & $2.68(-2.68,7.83)$ & 0.33 & $0.05(-10.1,11.3)$ & 0.99 \\
\hline MCPP & $-2.89(-5.65,-0.02)$ & $0.049 *$ & $-2.01(-6.12,2.10)$ & 0.34 & $1.60(-6.05,9.88)$ & 0.68 \\
\hline MCOP & $-2.03(-5.08,0.98)$ & 0.18 & $-1.11(-5.26,3.13)$ & 0.61 & $-2.52(-10.3,6.13)$ & 0.56 \\
\hline MCNP & $0.48(-2.03,2.94)$ & 0.71 & $-1.64(-5.28,1.93)$ & 0.36 & $3.50(-2.98,10.4)$ & 0.30 \\
\hline \multirow[t]{2}{*}{ ¿DEHP } & $-0.28(-3.63,0.30)$ & 0.87 & $-0.93(-5.56,3.79)$ & 0.70 & $7.21(-1.87,16.6)$ & 0.12 \\
\hline & \multicolumn{2}{|c|}{ Estradiol ( $n=175$ observations) } & \multicolumn{2}{|c|}{ SHBG ( $n=147$ observations) } & \multicolumn{2}{|c|}{ In-progesterone ( $n=179$ observations) } \\
\hline $\mathrm{MBzP}$ & $0.86(-8.52,10.3)$ & 0.86 & $4.89(-1.52,11.3)$ & 0.13 & $3.16(-4.56,11.5)$ & 0.67 \\
\hline MBP & $2.65(-5.90,11.2)$ & 0.54 & $-0.65(-6.29,4.98)$ & 0.82 & $3.71(-3.44,11.2)$ & 0.31 \\
\hline MiBP & $1.56(-6.27,9.39)$ & 0.69 & $1.19(-4.25,6.61)$ & 0.66 & $2.48(-3.96,9.49)$ & 0.46 \\
\hline MEP & $1.60(-8.49,11.7)$ & 0.75 & $-2.24(-9.43,4.97)$ & 0.54 & $-10.6(-17.6,-2.84)$ & $0.01^{*}$ \\
\hline MCPP & $-3.86(-11.5,3.80)$ & 0.32 & $-1.27(-6.40,3.86)$ & 0.62 & $-4.31(-10.2,1.98)$ & 0.17 \\
\hline MCOP & $-3.59(-11.4,4.24)$ & 0.36 & $-0.93(-6.44,4.58)$ & 0.74 & $-4.83(-11.0,1.57)$ & 0.13 \\
\hline MCNP & $-2.03(-8.50,4.41)$ & 0.53 & $-0.66(-4.85,3.46)$ & 0.75 & $-2.06(-7.22,3.28)$ & 0.43 \\
\hline$\Sigma \mathrm{DEHP}$ & $-0.56(-9.17,8.06)$ & 0.90 & $-4.11(-9.83,1.62)$ & 0.16 & $1.79(-5.17,9.39)$ & 0.62 \\
\hline
\end{tabular}

$\mathrm{Cl}$, confidence interval, TSH, thyroid-stimulating hormone, SHBG, sex hormone-binding globulin. Linear mixed models include a random intercept for subject ID and are adjusted for age at enrollment, prepregnancy body mass index, as well as urinary specific gravity and study visit. ${ }^{*} \mathrm{P}<0.05$. 
Table 5 Cross-sectional analysis: percent change $(95 \% \mathrm{Cls})$ in thyroid hormone concentrations in relation to interquartile range increase in urinary phthalate metabolite concentration by visit during gestation

\begin{tabular}{|c|c|c|c|c|c|c|}
\hline \multicolumn{7}{|c|}{ Visit 1 (16-20 weeks) } \\
\hline \multirow[t]{2}{*}{ Analyte } & $\%$ Change $(95 \% \mathrm{Cl})$ & p-value & $\%$ Change $(95 \% \mathrm{Cl})$ & p-value & $\%$ Change $(95 \% \mathrm{Cl})$ & p-value \\
\hline & \multicolumn{2}{|c|}{ Free T3 ( $n=100$ observations) } & \multicolumn{2}{|c|}{ Free T4 ( $n=100$ observations) } & \multicolumn{2}{|c|}{ In-TSH ( $n=100$ observations) } \\
\hline $\mathrm{MBzP}$ & $1.87(-2.55,6.37)$ & 0.40 & $2.44(-2.77,7.65)$ & 0.36 & $12.9(-0.93,28.2)$ & $0.07+$ \\
\hline MBP & $-0.72(-4.94,3.29)$ & 0.73 & $0.26(-4.58,5.04)$ & 0.92 & $1.29(-10.2,13.7)$ & 0.83 \\
\hline MiBP & $-1.64(-5.55,2.42)$ & 0.43 & $4.95(0.27,9.28)$ & $0.04^{*}$ & $1.09(-10.3,13.9)$ & 0.86 \\
\hline MEP & $-0.20(-5.37,5.02)$ & 0.94 & $-0.05(-6.10,6.10)$ & 0.99 & $0.77(-13.6,17.7)$ & 0.92 \\
\hline MCPP & $-1.04(-4.54,2.62)$ & 0.57 & $1.41(-2.83,5.80)$ & 0.50 & $-2.57(-12.7,8.69)$ & 0.64 \\
\hline MCOP & $-0.50(-4.11,2.93)$ & 0.78 & $-0.44(-4.58,3.76)$ & 0.83 & $-0.39(-10.3,10.7)$ & 0.94 \\
\hline MCNP & $1.05(-2.27,4.49)$ & 0.53 & $0.43(-3.47,4.37)$ & 0.83 & $-2.68(-11.9,7.35)$ & 0.58 \\
\hline$\Sigma \mathrm{DEHP}$ & $-1.62(-6.05,2.85)$ & 0.47 & $3.09(-2.17,8.27)$ & 0.25 & $7.14(-6.12,22.3)$ & 0.30 \\
\hline \multicolumn{7}{|c|}{ Visit 3 (24-28 weeks) } \\
\hline & \multicolumn{2}{|c|}{ Free T3 ( $n=81$ observations) } & \multicolumn{2}{|c|}{ Free T4 ( $n=81$ observations) } & \multicolumn{2}{|c|}{ In-TSH ( $\mathrm{n}=81$ observations) } \\
\hline $\mathrm{MBzP}$ & $-2.67(-8.3,2.86)$ & 0.34 & $-2.97(-12.1,6.07)$ & 0.51 & $-4.62(-20.4,13.5)$ & 0.58 \\
\hline $\mathrm{MBP}$ & $-0.57(-5.68,4.34)$ & 0.82 & $-1.46(-9.67,6.74)$ & 0.72 & $9.57(-6.27,27.3)$ & 0.25 \\
\hline MiBP & $-2.51(-6.81,1.70)$ & 0.24 & $-4.70(-11.8,2.08)$ & 0.17 & $2.74(-9.94,17.9)$ & 0.68 \\
\hline MEP & $2.76(-2.68,8.44)$ & 0.32 & $4.93(-3.81,13.7)$ & 0.27 & $18.1(0.00,37.8)$ & $0.05+$ \\
\hline MCPP & $-5.93(-10.2,-1.75)$ & $0.01^{*}$ & $-5.11(-12.5,2.22)$ & 0.17 & $2.41(-11.1,17.7)$ & 0.74 \\
\hline MCOP & $-5.83(-10.8,-0.58)$ & $0.03^{*}$ & $-3.02(-11.5,5.38)$ & 0.47 & $-3.35(-17.1,13.6)$ & 0.67 \\
\hline MCNP & $-1.44(-5.92,3.10)$ & 0.54 & $-6.34(-14.0,0.99)$ & $0.09+$ & $5.39(-8.50,21.3)$ & 0.46 \\
\hline$\Sigma \mathrm{DEHP}$ & $0.05(-4.49,4.49)$ & 0.98 & $-8.02(-15.3,-0.80)$ & $0.03^{*}$ & $2.79(-10.8,18.6)$ & 0.70 \\
\hline
\end{tabular}

$\mathrm{Cl}$, confidence interval, TSH, thyroid-stimulating hormone. Linear regression models adjusted for age at enrollment, prepregnancy body mass index, and urinary specific gravity.

${ }^{*} \mathrm{P}<0.05$.

$+\mathrm{P}<0.10$.

endocrine-disrupting effect of phthalates may depend on the timing of exposure during gestation.

Of the thyroid hormones, we observed that FT4 was inversely associated with certain urinary phthalate metabolites at visit 3 ( $26 \pm 2$ weeks of gestation). These results are in contrast to a previous cross-sectional investigation conducted among 76 Taiwanese pregnant women with urinary phthalate metabolites and hormone levels measured in the second trimester (mean time of sample collection $=27.9 \pm 2.3$ weeks of gestation) [23]. Huang and colleagues reported a statistically significant inverse association between urinary MBP and serum FT4 after adjusting for similar covariates in their regression model. We did not observe an analogous association at visit 3 (a comparable gestational age at sample collection) in the present study. FT3 was not measured in this previous investigation and regression results for TSH were not presented by the authors, thereby precluding additional comparisons with findings from our analyses. Differences between the results of our study and the Taiwanese study may be due to dissimilarities in study design, participant demographic characteristics, exclusion/inclusion criteria and/or exposures. For example, the median concentration (unadjusted for urinary dilution) of MBP was approximately four times higher in the Taiwanese pregnant women $(81.8 \mathrm{ng} / \mathrm{mL})$ [23] compared to the corresponding concentration found in women in the PROTECT cohort (20.1 ng/mL) [31].

Our findings for urinary metabolites of DEHP and serum FT4 and TSH are consistent with a previous study of adults (including non-pregnant women and men) participating in NHANES that reported significant inverse associations between certain urinary DEHP metabolites and FT4, although that larger study also reported positive relationships between these metabolites and TSH which were not significant in the present study [15]. Our results for FT3 and FT4 are also in agreement with the limited animal studies in which rats fed DEHPcontaminated diets, at doses orders of magnitude higher than those experienced by the PROTECT participants, had lower plasma T4 levels compared to controls and plasma T3 levels remained unchanged [42-45].

Various biological mechanisms have been proposed through which environmental chemicals may exert their action on thyroid function. Thyroid-disrupting chemicals may target the hypothalamic-pituitary-thyroid axis at multiple levels and may disrupt thyroid hormone homeostasis by interfering with the synthesis and regulation by the 
Table 6 Cross-sectional analysis: percent change (95\% Cls) in sex hormone concentrations in relation to interquartile range increase in urinary phthalate metabolite concentration by visit during gestation

\begin{tabular}{|c|c|c|c|c|c|c|}
\hline \multicolumn{7}{|c|}{ Visit 1 (16-20 weeks) } \\
\hline \multirow[t]{2}{*}{ Analyte } & $\%$ Change $(95 \% \mathrm{Cl})$ & p-value & $\%$ Change $(95 \% \mathrm{Cl})$ & p-value & $\%$ Change $(95 \% \mathrm{Cl})$ & p-value \\
\hline & \multicolumn{2}{|c|}{ Estradiol ( $n=97$ observations) } & \multicolumn{2}{|c|}{ SHBG ( $n=83$ observations) } & \multicolumn{2}{|c|}{ In-progesterone ( $\mathrm{n}=98$ observations) } \\
\hline $\mathrm{MBzP}$ & $-5.65(-18.9,7.57)$ & 0.40 & $4.99(-4.43,14.4)$ & 0.29 & $1.88(-6.60,11.3)$ & 0.66 \\
\hline MBP & $0.20(-10.3,14.2)$ & 0.75 & $4.13(-3.66,11.9)$ & 0.29 & $5.68(-2.41,13.7)$ & 0.18 \\
\hline MiBP & $-0.99(-13.1,11.1)$ & 0.87 & $-0.92(-9.77,7.28)$ & 0.77 & $5.56(-2.46,13.9)$ & 0.18 \\
\hline MEP & $0.03(-15.5,15.6)$ & 1.00 & $-10.1(-21.8,1.65)$ & $0.09+$ & $-12.15(-20.5,-2.99)$ & $0.01^{*}$ \\
\hline MCPP & $-2.93(-14.4,8.54)$ & 0.61 & $-2.66(-10.6,5.27)$ & 0.51 & $-0.42(-7.61,7.34)$ & 0.91 \\
\hline MCOP & $0.70(-9.86,11.3)$ & 0.90 & $0.86(-6.74,8.48)$ & 0.82 & $-0.57(-7.27,6.62)$ & 0.87 \\
\hline MCNP & $2.43(-7.42,12.3)$ & 0.63 & $-0.65(-7.75,6.45)$ & 0.86 & $-0.58(-6.85,6.11)$ & 0.86 \\
\hline$\Sigma \mathrm{DEHP}$ & $-7.50(-20.9,5.92)$ & 0.27 & $-5.93(-15.4,3.54)$ & 0.22 & $2.37(-6.23,11.9)$ & 0.59 \\
\hline \multicolumn{7}{|c|}{ Visit 3 (24-28 weeks) } \\
\hline & \multicolumn{2}{|c|}{ Estradiol ( $n=78$ observations) } & \multicolumn{2}{|c|}{ SHBG ( $n=64$ observations) } & \multicolumn{2}{|c|}{ In-progesterone ( $\mathrm{n}=\mathbf{8 1}$ observations) } \\
\hline $\mathrm{MBzP}$ & $4.38(-7.12,15.9)$ & 0.45 & $6.66(-4.98,18.3)$ & 0.26 & $8.02(-5.12,23.4)$ & 0.24 \\
\hline MBP & $6.55(-3.60,16.7)$ & 0.20 & $5.22(-5.18,15.7)$ & 0.32 & $8.19(-3.61,21.0)$ & 0.18 \\
\hline MiBP & $2.42(-5.96,10.8)$ & 0.57 & $3.46(-4.89,11.8)$ & 0.41 & $2.86(-6.81,13.8)$ & 0.58 \\
\hline MEP & $1.36(-9.57,12.3)$ & 0.81 & $1.00(-10.4,12.4)$ & 0.86 & $-9.43(-20.1,2.51)$ & 0.11 \\
\hline MCPP & $-0.48(-9.45,8.48)$ & 0.91 & $4.76(-3.69,13.2)$ & 0.26 & $-3.81(-11.8,6.87)$ & 0.47 \\
\hline MCOP & $-2.17(-12.4,8.09)$ & 0.67 & $3.93(-5.77,13.6)$ & 0.42 & $-5.15(-15.7,7.06)$ & 0.39 \\
\hline MCNP & $-2.64(-11.7,6.45)$ & 0.57 & $2.70(-6.31,11.7)$ & 0.55 & $-3.99(-13.9,6.75)$ & 0.45 \\
\hline$\Sigma \mathrm{DEHP}$ & $2.87(-6.17,11.9)$ & 0.53 & $1.14(-8.61,10.9)$ & 0.82 & $5.85(-4.81,17.6)$ & 0.29 \\
\hline
\end{tabular}

$\mathrm{Cl}$, confidence interval, SHBG, sex hormone-binding globulin. Linear regression models adjusted for age at enrollment, prepregnancy body mass index, and urinary specific gravity.

${ }^{*} \mathrm{P}<0.05$.

$+\mathrm{P}<0.10$.

hypothalamic-pituitary thyroid hormones (i.e., thyrotropinreleasing hormone $[\mathrm{TRH}]$ and TSH), binding of thyroid hormones to distributor proteins, cellular uptake mechanisms of thyroid hormones, metabolism of thyroid hormones by iodothyronine deiodinases, transcriptional activity of thyroid hormone receptors and/or receptor activation $[46,47]$. It has been suggested that phthalates may bind to thyroid hormone receptors, consequently activating or inhibiting thyroid hormone action [48-50], although data overtly demonstrating the binding of phthalates to thyroid receptors are lacking. Available experimental studies have provided some evidence for these potential mechanisms of thyroid disruption. In vitro studies have shown that phthalates may alter the sodium/ iodide symporter-mediated uptake of iodide by thyroid follicular cells [51,52], exhibit thyroid receptor antagonist activities [24,53,54], or displace thyroid hormones (e.g., T3) from distributor proteins [55]. Additionally, phthalates were found to alter the transcription of genes involved in the hypothalamic-pituitary-thyroid axis as well as the whole-body content of thyroid hormones in zebrafish [56].

Of the sex hormones, we observed a consistent inverse relationship between urinary MEP and serum progesterone across the two time points in pregnancy. In contrast to these findings, the previously described study by Huang et al. [23] reported no relationship between urinary MEP or other phthalate metabolites and progesterone during approximately the same sampling period in pregnancy as visit 3 in the current study. At visit 1 (18 \pm 2 weeks of gestation), we observed a marginally significant inverse relationship between urinary MEP and SHBG, although no association was observed at visit 3 . These results are in agreement with a previous study conducted among 1,377 pregnant Australian women, in which an inverse relationship between serum MEP and SHBG concentrations was observed early in pregnancy (18 weeks of gestation) [30]. Animal studies investigating the potential toxicity of phthalates on the female reproductive system are scarce, and have primarily examined effects of DEHP and DBP. These phthalates have been shown to reduce progesterone production in female rats in vivo and in rat granulosa cells in vitro [57-59]. However, we did not observe these relationships with metabolites of DEHP and DBP in the present analysis.

Our null findings for estradiol were comparable to those of previous epidemiologic studies conducted among pregnant women $[23,29]$. However, available toxicological data 
have shown that the ovary, in particular the granulosa cells of preovulatory follicles, may be the primary target site for DEHP [60]. Specifically, in vivo and in vitro studies of DEHP-treated female cycling rats have demonstrated that suppressed estradiol production may be the principal functional modification by DEHP [60-62]. These toxicology studies suggest that phthalates have the potential to induce alterations in steroidogenesis in female animals, although further research is needed to fully characterize the specific modes of action. Examining this association in a larger sample of pregnant women may enable identification of subtler relationships.

While the timing of exposure to endocrine disrupting chemicals during pregnancy has been shown to influence both the severity and onset of adverse developmental and reproductive outcomes [63], we are aware of no epidemiologic studies that have attempted to identify periods of susceptibility to phthalate-induced alterations in thyroid and sex steroid hormone levels in pregnant women. During the first trimester, the fetus relies solely on maternal T3 and T4 until the fetal thyroid gland fully develops at approximately 10 weeks of gestation [64]. Thus, maintaining maternal euthyroidism during the first trimester is critical - even slight alterations in maternal thyroid hormones during this period of gestation has been associated with deleterious neurodevelopmental and reproductive outcomes [65-67]. In later pregnancy, maternal thyroid hormones are essential for fetal thyroid homeostasis [68]. Although we detected a significant inverse relationship between urinary MiBP and serum FT4 in the first trimester (i.e., at visit 1), the strongest findings for FT4 were observed in the second half of pregnancy (i.e., at visit 3). Despite epidemiological investigations that have shown the potential adverse reproductive consequences of overt thyroid disease in early and late gestation $[69,70]$, the impact of trimester-specific subclinical alterations in maternal thyroid function on pregnancy outcomes remains largely understudied. Furthermore, we observed a consistent inverse association between urinary MEP and progesterone at each study visit, and this effect was greatest earlier in pregnancy (i.e., visit 1). Because progesterone plays an essential physiological role in the establishment and maintenance of pregnancy, insufficient concentrations of this hormone throughout gestation may lead to pregnancy loss or preterm birth, depending on the timing of hormonal disruption $[12,71,72]$.

While our findings may have important public health implications, our study has several limitations. Characteristic of most preliminary analyses in an ongoing prospective cohort, our study was limited by a small sample size. We expected that the availability of multiple measurements per subject would increase the power to detect associations, but analysis revealed that the effects of phthalates on hormone disruption may be different depending on timing of exposure. Furthermore, given the large number of statistical tests performed in the present analyses, there is possibility of type- 1 error. Another potential limitation is our timing of data collection during mid-pregnancy, which may potentially bias our results since women who spontaneously aborted in the first trimester are not captured in our analyses. We do not have biological data from these women pertaining to the exposure and outcomes of interest to assess the direction of the potential bias. Our study may also have been limited by our evaluation of circulating thyroid hormones as a sole indicator of thyroid toxicity [73]. Measurements of peripheral thyroid hormones may not fully capture the phthalate-induced effects on thyroid homeostasis [68]. That is, blood levels of thyroid hormones may not correspond with actions at the receptor, such as regulation of gene expression and the developmental processes in which they are involved [73,74]. However, given the limited amount of data on this subject and the infeasibility of collecting more specific markers during pregnancy, levels of circulating thyroid hormone measurements may serve as the most appropriate biomarker of thyroid disruption in pregnant women. Additional studies are necessary to address potential phthalate-induced alterations in thyroid-hormone responsive genes relevant to pregnancy outcomes. It may also be possible that the serum measurements included in our analyses represented transient changes in thyroid and sex hormone levels that may not have persisted outside of the two measurement time points. However, even temporary and/or subclinical alterations in maternal hormone levels may be biologically relevant and may induce permanent effects on parturition and/or the developing fetus [68]. Our study was also limited by the lack of information concerning the iodine and selenium status of our study participants, which may be important because deficiencies in these trace elements can impair normal thyroid hormone function [75]. However, we have no reason to expect that these would be associated with phthalate exposure, so any deficiencies in these substances would affect the precision of the effect estimates rather than the effect estimates themselves [76]. Finally, our study was conducted among pregnant women in Northern Puerto Rico, which may have implications for the generalizability of results.

Despite these limitations, our study had several strengths. The collection of biomarker measurements at two time points during pregnancy enabled us to utilize mixed modeling techniques to more powerfully detect associations among repeated measurements and also evaluate potential periods of gestation during which phthalates may have a more profound impact on maternal hormone levels. Also, as pregnancy is characterized by a dynamic interplay between maternal endocrine hormones, our measurements of hormone concentrations from two 
disparate endocrine axes is advantageous. Each set of hormones may represent distinct mechanisms through which phthalates may influence downstream reproductive health outcomes. Furthermore, we used novel and precise analytical techniques to measure serum levels of FT3 and FT4. Especially pertinent to a population of pregnant women, this method has advantages over traditional immunoassays because it is more specific, does not crossreact with other analytes, and is not influenced by serum binding proteins $[35,77,78]$. Notably, thyroxine binding proteins may increase as much as 50 percent during pregnancy [78].

\section{Conclusions}

The results of our study provide suggestive evidence for phthalate-induced endocrinal disturbances during pregnancy, and may augment mechanistic understanding of the impact of phthalates on reproductive health outcomes. Future research on the specific pathways through which phthalates may alter thyroid and sex hormone concentrations are required for targeted interventions aimed at preventing downstream hormone-mediated adverse reproductive health outcomes in pregnant women.

\section{Additional files}

Additional file 1: Table S1. Urinary phthalate metabolite concentrations $(\mathrm{ng} / \mathrm{mL})$ in pregnant women from Puerto Rico $(\mathrm{N}=106)$.

Additional file 2: Table S2. Pearson correlations between serum sex hormones, serum thyroid hormones, and maternal age in pregnant women from Puerto Rico $(\mathrm{N}=106)$.

\section{Competing interests}

The authors declare that they have no competing interests.

\section{Authors' contributions}

$\sqcup$ performed the statistical analyses and drafted the manuscript. KF participated in the design of the study, assisted in the statistical analyses, and critically revised the manuscript. OS carried out the immunoassays for the thyroid and sex hormones. DC and LRG provided technical support and critically revised the manuscript. LT was involved in recruitment of study participants and collection of data. AC and XY carried out the laboratory analyses pertaining to the measurement of urinary phthalate metabolites and critically revised the manuscript. $\mathrm{AA}$ and $\mathrm{JC}$ participated in the coordination of the study and critically revised the manuscript. JM conceived the study, assisted in drafting the manuscript, and provided conceptual advice. All authors read and approved the final manuscript.

\section{Acknowledgements}

Work supported by P42ES017198, R01ES018872, P30ES017885, and T32ES007062 from the National Institute of Environmental Health Sciences (NIEHS). The content is solely the responsibility of the authors and does not necessarily represent the official views of the National Institute of Environmental Health Sciences, the National Institutes of Health, or the Centers for Disease Control and Prevention. We gratefully acknowledge Manori Silva, Ella Samandar, Jim Preau, and Tao Jia for technical assistance in measuring the urinary concentrations of the phthalate metabolites.

\section{Author details}

${ }^{1}$ Department of Environmental Health Sciences, University of Michigan School of Public Health, Ann Arbor, MI, USA. ²Department of Medicine, Georgetown University, Washington, DC, USA. ${ }^{3}$ Department of Obstetrics and
Gynecology, Brigham and Women's Hospital, Boston, MA, USA. ${ }^{4}$ University of Puerto Rico Graduate School of Public Health, UPR Medical Sciences Campus, San Juan, Puerto Rico. ${ }^{5}$ Centers for Disease and Control and Prevention, Atlanta, GA, USA. ${ }^{6}$ College of Engineering, Northeastern University, Boston, MA, USA.

Received: 4 September 2014 Accepted: 12 January 2015 Published: 17 January 2015

\section{References}

1. Swan SH. Environmental phthalate exposure in relation to reproductive outcomes and other health endpoints in humans. Environ Res. 2008;108:177-84.

2. Schettler T. Human exposure to phthalates via consumer products. Int J Androl. 2006;29:134-9. discussion 181-135.

3. Adibi JJ, Perera FP, Jedrychowski W, Camann DE, Barr D, Jacek R, et al. Prenatal exposures to phthalates among women in New York City and Krakow, Poland. Environ Health Perspect. 2003;111:1719-22.

4. Agency for Toxic Substances and Disease Registry (ATSDR). Toxicological profile for Di(2-ethylhexyl)phthalate (DEHP). City: U.S. Department of Health and Human Services, Public Health Service; 2002.

5. Meeker JD, Sathyanarayana S, Swan SH. Phthalates and other additives in plastics: human exposure and associated health outcomes. Philos Trans R Soc Lond B Biol Sci. 2009;364:2097-113.

6. Hauser R, Calafat AM. Phthalates and human health. Occup Environ Med. 2005;62:806-18.

7. Fromme H, Bolte G, Koch HM, Angerer J, Boehmer S, Drexler H, et al. Occurrence and daily variation of phthalate metabolites in the urine of an adult population. Int J Hyg Environ Health. 2007;210:21-33.

8. Ferguson KK, McElrath TF, Meeker JD. Environmental phthalate exposure and preterm birth. JAMA Pediatr. 2014;168:61-7.

9. Toft $G$, Jonsson $B A$, Lindh $C H$, Jensen $T K$, Hjollund $N H$, Vested $A$, et al. Association between pregnancy loss and urinary phthalate levels around the time of conception. Environ Health Perspect. 2012;120:458-63.

10. Robins JC, Marsit CJ, Padbury JF, Sharma SS. Endocrine disruptors, environmental oxygen, epigenetics and pregnancy. Front Biosci (Elite Ed). 2011;3:690-700.

11. Monticone S, AuchuS RJ, Rainey WE. Adrenal disorders in pregnancy. Nat Rev Endocrinol. 2012;8:668-78.

12. Mesiano S. In: Jerome F. Strauss III and Robert L. Barbieri, editors. Yen \& Jaffe's reproductive endocrinology: physiology, pathophysiology, and clinical management. 7th ed. Philadelphia, PA: Elsevier Saunders; 2013.

13. Haddow JE, Palomaki GE, Allan WC, Williams JR, Knight GJ, Gagnon J, et al. Maternal thyroid deficiency during pregnancy and subsequent neuropsychological development of the child. N Engl J Med. 1999:341:549-55.

14. Meeker JD, Calafat AM, Hauser R. Di(2-ethylhexyl) phthalate metabolites may alter thyroid hormone levels in men. Environ Health Perspect. 2007;115:1029-34

15. Meeker JD, Ferguson KK. Relationship between urinary phthalate and bisphenol A concentrations and serum thyroid measures in U.S. adults and adolescents from the National Health and Nutrition Examination Survey (NHANES) 2007-2008. Environ Health Perspect. 2011;119:1396-402.

16. Boas M, Frederiksen H, Feldt-Rasmussen U, Skakkebaek NE, Hegedus L, Hilsted $L$, et al. Childhood exposure to phthalates: associations with thyroid function, insulin-like growth factor I, and growth. Environ Health Perspect. 2010;118:1458-64.

17. Philippat C, Mortamais M, Chevrier C, Petit C, Calafat AM, Ye X, et al. Exposure to phthalates and phenols during pregnancy and offspring size at birth. Environ Health Perspect. 2012;120:464-70.

18. Casas L, Fernandez MF, Llop S, Guxens M, Ballester F, Olea N, et al. Urinary concentrations of phthalates and phenols in a population of Spanish pregnant women and children. Environ Int. 2011;37:858-66.

19. Meeker JD, Hu H, Cantonwine DE, Lamadrid-Figueroa H, Calafat AM, Ettinger $\mathrm{AS}$, et al. Urinary phthalate metabolites in relation to preterm birth in Mexico city. Environ Health Perspect. 2009;117:1587-92.

20. Irvin EA, Calafat AM, Silva MJ, Aguilar-Villalobos M, Needham LL, Hall DB, et al. An estimate of phthalate exposure among pregnant women living in Trujillo, Peru. Chemosphere. 2010;80:1301-7.

21. Lin S, Ku HY, Su PH, Chen JW, Huang PC, Angerer J, et al. Phthalate exposure in pregnant women and their children in central Taiwan. Chemosphere. 2011;82:947-55. 
22. Berman T, Hochner-Celnikier D, Calafat AM, Needham LL, Amitai Y, Wormser U, et al. Phthalate exposure among pregnant women in Jerusalem, Israel: results of a pilot study. Environ Int. 2009;35:353-7.

23. Huang PC, Kuo PL, Guo YL, Liao PC, Lee CC. Associations between urinary phthalate monoesters and thyroid hormones in pregnant women. Hum Reprod. 2007;22:2715-22.

24. Ghisari M, Bonefeld-Jorgensen EC. Effects of plasticizers and their mixtures on estrogen receptor and thyroid hormone functions. Toxicol Lett. 2009;189:67-77.

25. Craig ZR, Wang W, Flaws JA. Endocrine-disrupting chemicals in ovarian function: effects on steroidogenesis, metabolism and nuclear receptor signaling. Reproduction. 2011;142:633-46.

26. Feldt-Rasmussen U, Mathiesen ER. Endocrine disorders in pregnancy: physiological and hormonal aspects of pregnancy. Best Pract Res Clin Endocrinol Metab. 2011;25:875-84.

27. Meeker JD, Calafat AM, Hauser R. Urinary metabolites of di(2-ethylhexyl) phthalate are associated with decreased steroid hormone levels in adult men. J Androl. 2009;30:287-97.

28. Mendiola J, Jorgensen N, Andersson AM, Calafat AM, Silva MJ, Redmon JB, et al. Associations between urinary metabolites of di(2-ethylhexyl) phthalate and reproductive hormones in fertile men. Int J Androl. 2011:34:369-78.

29. Sathyanarayana S, Barrett E, Butts S, Wang C, Swan SH. Phthalate exposure and reproductive hormone concentrations in pregnancy. Reproduction. 2014;147:401-9.

30. Hart R, Doherty DA, Frederiksen H, Keelan JA, Hickey M, Sloboda D, et al. The influence of antenatal exposure to phthalates on subsequent female reproductive development in adolescence: a pilot study. Reproduction. 2014;147:379-90.

31. Cantonwine DE, Cordero JF, Rivera-Gonzalez LO, Anzalota Del Toro LV, Ferguson KK, Mukherjee B, et al. Urinary phthalate metabolite concentrations among pregnant women in Northern Puerto Rico: distribution, temporal variability, and predictors. Environ Int. 2014:62:1-11.

32. Silva MJ, Slakman AR, Reidy JA, Preau Jr JL, Herbert AR, Samandar E, et al. Analysis of human urine for fifteen phthalate metabolites using automated solid-phase extraction. J Chromatogr B Analyt Technol Biomed Life Sci. 2004;805:161-7.

33. Hornung RWR, L. D. Estimation of average concentration in the presence of nondetectable values. Appl Occup Environ Hyg. 1990;5:46-51.

34. Soldin SJ, Soukhova N, Janicic N, Jonklaas J, Soldin OP. The measurement of free thyroxine by isotope dilution tandem mass spectrometry. Clin Chim Acta. 2005;358:113-8.

35. Kahric-Janicic N, Soldin SJ, Soldin OP, West T, Gu J, Jonklaas J. Tandem mass spectrometry improves the accuracy of free thyroxine measurements during pregnancy. Thyroid. 2007;17:303-11.

36. Gu J, Soldin OP, Soldin SJ. Simultaneous quantification of free triiodothyronine and free thyroxine by isotope dilution tandem mass spectrometry. Clin Biochem. 2007:40:1386-91.

37. van Deventer HE, Mendu DR, Remaley AT, Soldin SJ. Inverse log-linear relationship between thyroid-stimulating hormone and free thyroxine measured by direct analog immunoassay and tandem mass spectrometry. Clin Chem. 2011;57:122-7.

38. Hatch EE, Nelson JW, Qureshi MM, Weinberg J, Moore LL, Singer M, et al. Association of urinary phthalate metabolite concentrations with body mass index and waist circumference: a cross-sectional study of NHANES data, 1999-2002. Environ Health. 2008;7:27.

39. lacobellis G, Ribaudo MC, Zappaterreno A, lannucci CV, Leonetti F. Relationship of thyroid function with body mass index, leptin, insulin sensitivity and adiponectin in euthyroid obese women. Clin Endocrinol (Oxf). 2005;62:487-91.

40. Ukkola O, Gagnon J, Rankinen T, Thompson PA, Hong Y, Leon AS, et al. Age, body mass index, race and other determinants of steroid hormone variability: the HERITAGE Family Study. Eur J Endocrinol. 2001;145:1-9.

41. Peeters RP. Thyroid hormones and aging. Hormones (Athens). 2008;7:28-35.

42. Hinton RH, Mitchell FE, Mann A, Chescoe D, Price SC, Nunn A, et al. Effects of phthalic acid esters on the liver and thyroid. Environ Health Perspect. 1986;70:195-210

43. Howarth JA, Price SC, Dobrota M, Kentish PA, Hinton RH. Effects on male rats of di-(2-ethylhexyl) phthalate and di-n-hexylphthalate administered alone or in combination. Toxicol Lett. 2001;121:35-43.

44. Poon R, Lecavalier P, Mueller R, Valli VE, Procter BG, Chu I. Subchronic oral toxicity of di-n-octyl phthalate and di(2-Ethylhexyl) phthalate in the rat. Food Chem Toxicol. 1997;35:225-39.
45. Price SC, Chescoe D, Grasso P, Wright M, Hinton RH. Alterations in the thyroids of rats treated for long periods with di-(2-ethylhexyl) phthalate or with hypolipidaemic agents. Toxicol Lett. 1988;40:37-46.

46. Jugan ML, Levi Y, Blondeau JP. Endocrine disruptors and thyroid hormone physiology. Biochem Pharmacol. 2010;79:939-47.

47. Boas M, Feldt-Rasmussen U, Main KM. Thyroid effects of endocrine disrupting chemicals. Mol Cell Endocrinol. 2012;355:240-8.

48. Zoeller RT. Environmental chemicals as thyroid hormone analogues: new studies indicate that thyroid hormone receptors are targets of industrial chemicals? Mol Cell Endocrinol. 2005;242:10-5.

49. Zoeller RT. Environmental chemicals impacting the thyroid: targets and consequences. Thyroid. 2007;17:811-7.

50. Kashiwagi K, Furuno N, Kitamura S, Ohta S, Sugihara K, Utsumi K, et al. Disruption of Thyroid Hormone Function by Environmental Pollutants. J Health Sci. 2009;55:147-60.

51. Wenzel A, Franz C, Breous E, Loos U. Modulation of iodide uptake by dialkyl phthalate plasticisers in FRTL-5 rat thyroid follicular cells. Mol Cell Endocrinol. 2005;244:63-71.

52. Breous $\mathrm{E}$, Wenzel $\mathrm{A}$, Loos $\mathrm{U}$. The promoter of the human sodium/iodide symporter responds to certain phthalate plasticisers. Mol Cell Endocrinol. 2005;244:75-8

53. Sugiyama S, Shimada N, Miyoshi H, Yamauchi K. Detection of thyroid system-disrupting chemicals using in vitro and in vivo screening assays in Xenopus laevis. Toxicol Sci. 2005;88:367-74.

54. Shen $\mathrm{O}, \mathrm{Du} G$, Sun $H$, Wu W, Jiang $Y$, Song $L$, et al. Comparison of in vitro hormone activities of selected phthalates using reporter gene assays. Toxicol Lett. 2009;191:9-14.

55. Ishihara A, Sawatsubashi S, Yamauchi K. Endocrine disrupting chemicals: interference of thyroid hormone binding to transthyretins and to thyroid hormone receptors. Mol Cell Endocrinol. 2003;199:105-17.

56. Zhai W, Huang Z, Chen L, Feng C, Li B, Li T. Thyroid endocrine disruption in zebrafish larvae after exposure to mono-(2-ethylhexyl) phthalate (MEHP). PLoS One. 2014;9:e92465

57. Svechnikova I, Svechnikov K, Soder O. The influence of di-(2-ethylhexyl) phthalate on steroidogenesis by the ovarian granulosa cells of immature female rats. J Endocrinol. 2007;194:603-9.

58. Treinen KA, Dodson WC, Heindel JJ. Inhibition of FSH-stimulated cAMP accumulation and progesterone production by mono(2-ethylhexyl) phthalate in rat granulosa cell cultures. Toxicol Appl Pharmacol. 1990;106:334-40.

59. Gray Jr LE, Laskey J, Ostby J. Chronic di-n-butyl phthalate exposure in rats reduces fertility and alters ovarian function during pregnancy in female Long Evans hooded rats. Toxicol Sci. 2006;93:189-95.

60. Lovekamp-Swan T, Davis BJ. Mechanisms of phthalate ester toxicity in the female reproductive system. Environ Health Perspect. 2003;111:139-45.

61. Davis BJ, Maronpot RR, Heindel JJ. Di-(2-ethylhexyl) phthalate suppresses estradiol and ovulation in cycling rats. Toxicol Appl Pharmacol. 1994;128:216-23.

62. Laskey JW, Berman E. Steroidogenic assessment using ovary culture in cycling rats: effects of bis(2-diethylhexyl)phthalate on ovarian steroid production. Reprod Toxicol. 1993;7:25-33.

63. Marques-Pinto A, Carvalho D. Human infertility: are endocrine disruptors to blame? Endocr Connect. 2013;2:R15-29.

64. Glinoer D, de Nayer P, Bourdoux P, Lemone M, Robyn C, van Steirteghem A, et al. Regulation of maternal thyroid during pregnancy. J Clin Endocrinol Metab. 1990;71:276-87.

65. Krassas GE, Poppe K, Glinoer D. Thyroid function and human reproductive health. Endocr Rev. 2010;31:702-55.

66. Stagnaro-Green A, Abalovich M, Alexander E, Azizi F, Mestman J, Negro R, et al. Guidelines of the American Thyroid Association for the diagnosis and management of thyroid disease during pregnancy and postpartum. Thyroid. 2011:21:1081-125.

67. Casey BM, Dashe JS, Wells CE, McIntire DD, Byrd W, Leveno K, et al. Subclinical hypothyroidism and pregnancy outcomes. Obstet Gynecol. 2005;105:239-45

68. Hartoft-Nielsen ML, Boas M, Bliddal S, Rasmussen AK, Main K, Feldt-Rasmussen U. Do thyroid disrupting chemicals influence foetal development during pregnancy? J Thyroid Res. 2011;2011:342189.

69. Idris I, Srinivasan R, Simm A, Page RC. Maternal hypothyroidism in early and late gestation: effects on neonatal and obstetric outcome. Clin Endocrinol (Oxf). 2005;63:560-5. 
70. Phoojaroenchanachai M, Sriussadaporn S, Peerapatdit T, Vannasaeng S, Nitiyanant W, Boonnamsiri V, et al. Effect of maternal hyperthyroidism during late pregnancy on the risk of neonatal low birth weight. Clin Endocrinol (Oxf). 2001;54:365-70.

71. Ozlu T, Gungor AC, Donmez ME, Duran B. Use of progestogens in pregnant and infertile patients. Arch Gynecol Obstet. 2012;286:495-503.

72. Young SL, Lessey BA. Progesterone function in human endometrium: clinical perspectives. Semin Reprod Med. 2010;28:5-16.

73. Zoeller TR, Dowling AL, Herzig CT, lannacone EA, Gauger K, Bansal R. Thyroid hormone, brain development, and the environment. Environ Health Perspect. 2002;110 Suppl 3:355-61.

74. Massart F, Ferrara P, Saggese G. Environmental thyroid disruptors and human endocrine health. In: A New look at hypothyroidism. New York: Springer D: InTech; 2012.

75. Zimmermann MB, Kohrle J. The impact of iron and selenium deficiencies on iodine and thyroid metabolism: biochemistry and relevance to public health. Thyroid. 2002:12:867-78

76. Schisterman EF, Cole SR, Platt RW. Overadjustment bias and unnecessary adjustment in epidemiologic studies. Epidemiology. 2009;20:488-95.

77. Soldin OP, Tractenberg RE, Hollowell JG, Jonklaas J, Janicic N, Soldin SJ Trimester-specific changes in maternal thyroid hormone, thyrotropin, and thyroglobulin concentrations during gestation: trends and associations across trimesters in iodine sufficiency. Thyroid. 2004;14:1084-90.

78. Soldin OP, Soldin SJ. Thyroid hormone testing by tandem mass spectrometry. Clin Biochem. 2011;44:89-94.

doi:10.1186/1477-7827-13-4

Cite this article as: Johns et al:: Urinary phthalate metabolites in relation to maternal serum thyroid and sex hormone levels during pregnancy: a longitudinal analysis. Reproductive Biology and Endocrinology 2015 13:4.

\section{Submit your next manuscript to BioMed Central and take full advantage of:}

- Convenient online submission

- Thorough peer review

- No space constraints or color figure charges

- Immediate publication on acceptance

- Inclusion in PubMed, CAS, Scopus and Google Scholar

- Research which is freely available for redistribution 